\title{
Illustration of a number of atypical computed tomography manifestations of active pulmonary tuberculosis
}

\author{
Yi Zeng ${ }^{1 \#}$, Xiao-Li Zhai ${ }^{2 \#}$, Yì Xiáng J. Wáng ${ }^{3}$, Wei-Wei Gao ${ }^{1}$, Chun-Mei Hu ${ }^{1}$, Fei-Shen Lin ${ }^{1}$, \\ Wen-Shu Chai ${ }^{4}$, Jian-Yun Wang ${ }^{5}$, Yan-Ling Shi ${ }^{6}$, Xin-Hua Zhou ${ }^{7}$, Hui-Shan Yu ${ }^{8}$, Xi-Wei Lu ${ }^{9}$ \\ ${ }^{1}$ Department of Tuberculosis, Nanjing Public Health Medical Center, Nanjing Second Hospital, Nanjing Hospital Affiliated to Nanjing University of \\ Traditional Chinese Medicine, Nanjing, China; ${ }^{2}$ Department of Radiology, Beijing Chaoyang Hospital, Capital Medical University, Beijing, China; \\ ${ }^{3}$ Department of Imaging and Interventional Radiology, The Chinese University of Hong Kong, Hong Kong, China; ${ }^{4}$ Department of Respiratory \\ Medicine, First Affiliated Hospital of Jinzhou Medical University, Jinzhou, China; ${ }^{5}$ Department of Radiology, Lanzhou Lung Hospital, Lanzhou, \\ China; ${ }^{6}$ Department of Radiology, Second Hospital of Daqing City, Daqing, China; ${ }^{7}$ Department of Radiology, Beijing Chest Hospital, Capital \\ Medical University, Beijing, China; ${ }^{8}$ Department of Radiology, Wuhan Pulmonary Hospital, Wuhan, China; ${ }^{9}$ Department of Tuberculosis, Dalian \\ Tuberculosis Hospital, Dalian, China
}

\#These authors contributed equally to this work.

Correspondence to: Dr. Xi-Wei Lu. Department of Tuberculosis, Dalian Tuberculosis Hospital, 277 Juniper Road, Ganjingzi District, Dalian 116033, China. Email: yiluxiwei@126.com.

\begin{abstract}
Tuberculosis is a serious public health challenge facing mankind and one of the top ten causes of death. Diagnostic imaging plays an important role, particularly for the diagnosis and treatment planning of tuberculosis patients with negative microbiology results. This article illustrates a number of atypical computed tomography (CT) appearances of pulmonary tuberculosis (PTB), including (I) clustered micronodules (CMNs) sign; (II) reversed halo sign (RHS); (III) tuberculous pneumatocele; (IV) hematogenously disseminated PTB with predominantly diffuse ground glass opacity manifestation; (V) hematogenously disseminated PTB with randomly distributed non-miliary nodules; (VI) PTB changes occur on the background of emphysema or honeycomb changes of interstitial pneumonia; and (VII) PTB manifesting as organizing pneumonia. While the overall incidence of PTB is decreasing globally, the incidence of atypical manifestations of tuberculosis is increasing. A good understanding of the atypical CT imaging changes of active PTB shall help the diagnosis and differential diagnosis of PTB in clinical practice.
\end{abstract}

Keywords: Pulmonary tuberculosis (PTB); sarcoid galaxy sign; miliary tuberculosis; cluster of micronodules; reversed halo sign (RHS); emphysema; pneumatocele; interstitial pneumonia; organizing pneumonia (OP)

Submitted Dec 01, 2020. Accepted for publication Dec 28, 2020.

doi: $10.21037 /$ qims-20-1323

View this article at: http://dx.doi.org/10.21037/qims-20-1323

Tuberculosis is a serious public health challenge facing mankind and one of the top ten causes of death. In 2018, an estimated 10 million patients worldwide had new tuberculosis. And in 2018, among HIV-negative people, tuberculosis caused approximately 1.2 million deaths (compared to 1.7 million in 2000 , a decrease of $27 \%$ ). Among HIV-positive patients, 251,000 died of tuberculosis (compared to 620,000 in 2000 , down $60 \%$ ). $55 \%$ of pulmonary tuberculosis (PTB) cases have pathogenic evidence, while $45 \%$ are diagnosed through imaging and

^ ORCID: 0000-0001-5697-0717 

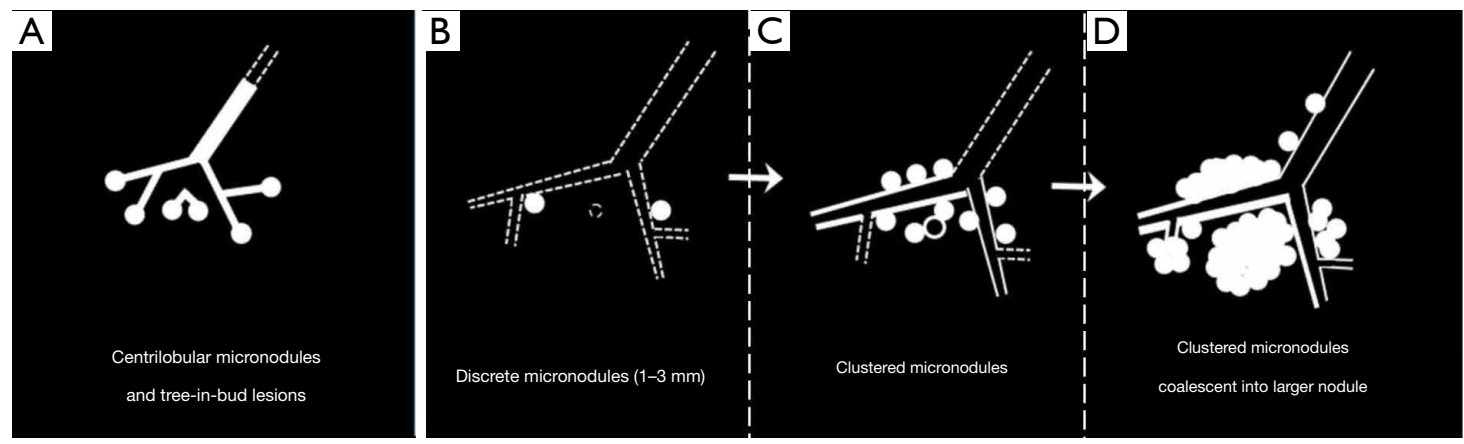

Figure 1 Diagrams showing characteristic findings of centrilobular micronodules and tree-in-bud lesions (A) and clustered micronodules $(\mathrm{CMNs})(\mathrm{B}, \mathrm{C}, \mathrm{D})$. Note that $\mathrm{CMNs}$ originate from a few discrete micronodules $(1-3 \mathrm{~mm})$ which primarily distribute around small airways distal to the level of segmental bronchus (B). Over time, localized aggregation of multiple discrete micronodules associated with small airway wall thickening and bronchiolectasis gradually progress to CMNs (C). Unlike tree-in-bud lesions which suggest small airways filled with caseous materials (A), the CMNs may have association with thickened walls of small airways maintaining luminal patency. The CMNs may merge into a larger nodule or consolidation either initially or later as CMNs progress and can obscure small airways (D). Dotted line: normal bronchiole; solid line: bronchiectasis; thickened solid line: bronchiectasis with bronchial wall thickening. Reproduced with permission from (6).

other non-pathogenic means (1).

It is expected that in the future imaging will continue to play an important role, particularly for the diagnosis and treatment planning of tuberculosis patients with negative microbiology results. Computed tomography (CT) signs of active PTB continue to be further recognized. The common CT signs of $\mathrm{PTB}$ include cavities, tree-in-buds, nodules, and lung consolidation. The findings of cavities, centrilobular micronodules, bronchial wall thickening, and tree-in-bud lesions on CT images usually represent caseation materials within or surrounding terminal or respiratory bronchioles and the alveolar duct, suggesting endobronchial spread of tuberculosis. In tuberculosis patients with diabetes, HIV positive status, and those with the use of hormones and immunosuppressants, CT features may not present typical characteristics (2-5).

Atypical tuberculosis is not a strict academic term, instead it is related to various uncertainties including symptoms, laboratory tests, as well as diagnostic imaging. Although the signs of atypical tuberculosis are not commonly encountered clinically, as long as the rules are understood, these atypical tuberculosis cases can be identified. Moreover, some atypical signs may only appear during a specific stage in the usual progression or regression of tuberculous pathology. This article illustrates a number of rare and often pathognomonic CT signs of PTB.

\section{Clustered micronodules (CMNs) manifestation of PTB}

CMNs is the sign of an aggregation of multiple discrete dense micronodules $(1-3 \mathrm{~mm})$ spaced apart of a few millimeters or less, which primarily distribute around small airways distal to the level of the segmental bronchus (Figure 1) (6). Small airways surrounded by $\mathrm{CMNs}$ usually maintain luminal patency and are often dilated with thickened walls. CMNs include micronodules in the peribronchiolar stroma with no branch line connection, and the airways in this area are usually unobstructed (while there may be thickening and dilation), and there is usually no shadow of the airway blocked by caseous necrosis. When the CMNs have a coalescence, small airways could be obscured by the conglomeration. When the CMNs merged to form large nodules or consolidation with the surrounding small nodules still remaining, thus resembling the Milky Way galaxy, this finding is named as galaxy sign.

The most common finding of bronchogenic spread of PTB on thin section CT is centrilobular nodules and branching linear structures, giving a tree-in-bud appearance. This finding is different in appearance from tuberculous CMNs, although both findings may coexist (6).

The CMN pattern of PTB mostly occurs in the upper and middle lung fields, and the nodules are clustered and arranged in patchy shapes, which can be segmentally or non- 


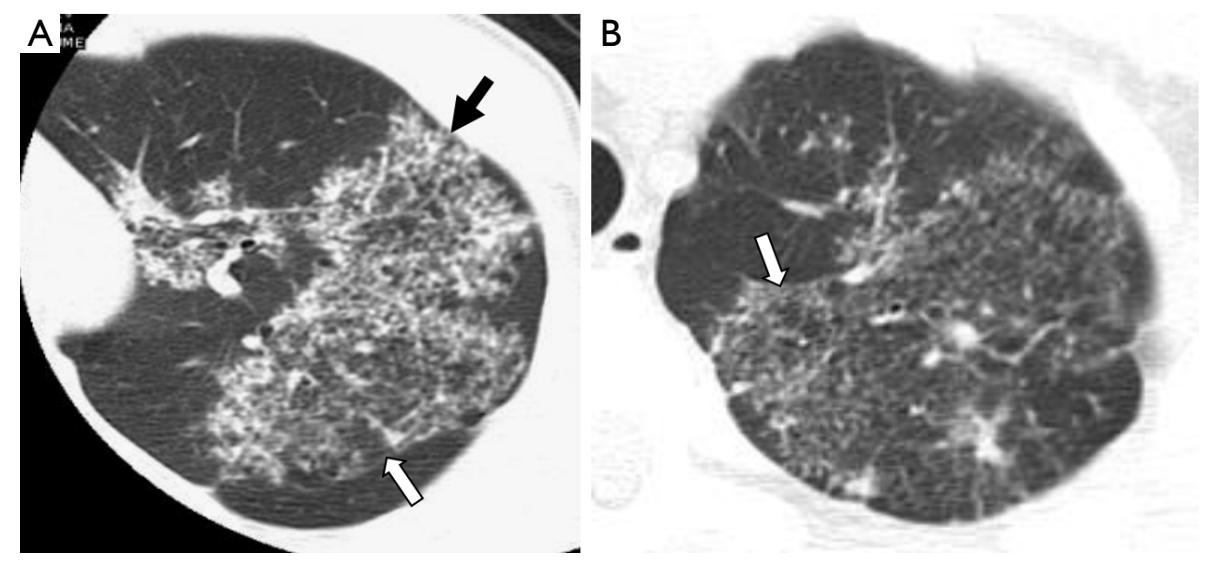

Figure 2 Clustered micronodules (CMNs) computed tomography (CT) manifestation of pulmonary tuberculosis. (A) A 31-year-old male patient with pulmonary tuberculosis. CMNs distributes in the left upper lobe, with reticular lines. The edge of the lesion is surrounded by a line shadow (white arrow), indicating the thickened lobular interval. This is called the "marginal sign". Moreover, tree-in-bud sign is seen at the edge of the lesion (black arrow). (B) A 24-year-old female patient with pulmonary tuberculosis. High resolution CT shows CMNs in the posterior segment of the left upper lobe, with clear linear edges visible (white arrow) on the front border of the lesions.
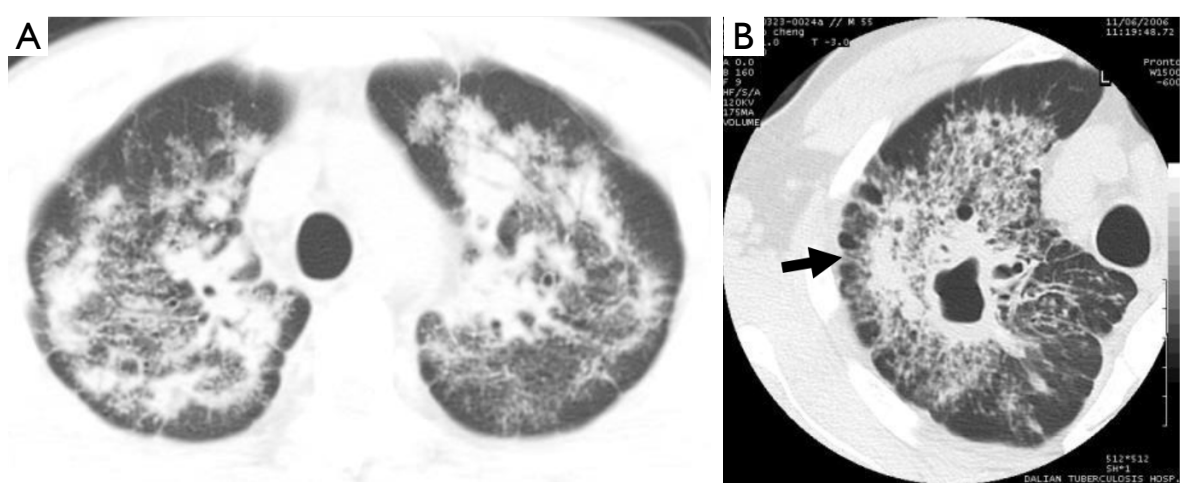

Figure 3 Clustered micronodules (CMNs) computed tomography (CT) manifestation of pulmonary tuberculosis. (A) A 31-year-old female patient with pulmonary tuberculosis. CMNs in both lungs partially merged to form lung consolidations. (B) A 55-year-old male patient with pulmonary tuberculosis and positive sputum microbiology. CT shows non-segmentally distributed cluster-like CMNs in the right upper lobe, with merged consolidation and a thick-walled cavity in the center as well as thickened and dilated bronchial walls. Thickening of the interlobular septum can be seen at the pleural surface of the lesions (black arrow).

segmentally distributed, and can be single or multiple. High resolution CT suggests that PTB CMNs is a combination of a group of signs, including cluster-like distribution of micro-nodules, with or without ground glass shadow, with tree-in-bud sign as an important accompanying sign around the CMN lesion (Figures 2-4). In the areas distributed with CMNs, fine grid-like shadows formed by thickening of the interstitium within the lobules can be observed. The thickening of the interlobular interval can be seen on the edge of some CMNs lesions, which surrounds or blocks the spread of micro-nodules, so that CMNs may have a clear boundary. This is called "marginal sign" (Figure 2). As the lesion progresses, this "marginal sign" disappears, and the central areas of the lesion can merge into consolidation, followed by caseous necrosis and cavity formation (Figure 3). For multiple CMNs, the morphological characteristics of the lesions can be roughly the same (Figure 4).

CMNs and galaxy sign can be seen in active PTB in about $8.4-16 \%$ cases (5-7). According to the study of Hong et al. (6) which included 833 patients, CMNs was the most 

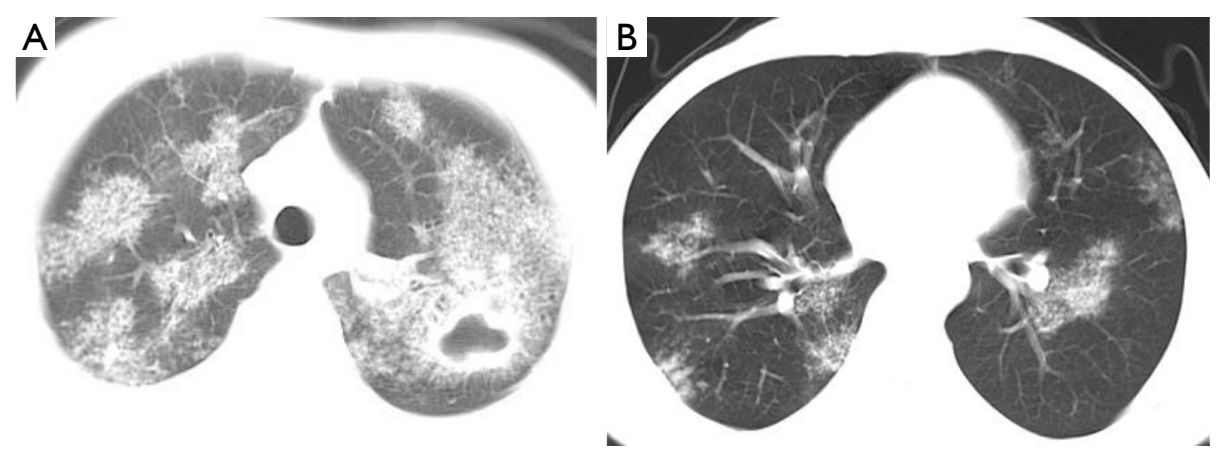

Figure 4 Clustered micronodules (CMNs) computed tomography (CT) manifestation of pulmonary tuberculosis. A 27-year-old female patient with pulmonary tuberculosis and positive sputum microbiology. CT showed multiple CMNs in both lungs, dominant in upper and middle lungs. Fusion and necrosis occurred in the CMNs area of the left upper lung, forming a thick-walled cavity (A). There are fewer lesions in the middle and lower lung fields (B), with the CMNs roughly the same in shape.

predominant diagnostic CT abnormality in $2.6 \%$ patients $(\mathrm{n}=22)$. Among the remaining 811 patients, 763 patients did not have CMNs, and CMNs presented as a minor CT abnormality in 48 patients $(5.9 \%)$. The apicoposterior segment of the left upper lobe was the most commonly involved segment (10/22, 45.5\%). Patients with predominant CMNs had a significantly lower rate of acid-fast bacilli smear positivity. However, positivity rates for PCR assays and culture may not differ from other PTB cases (6).

CMNs is more likely to be a well-confined and indolently progressing pulmonary parenchymal tuberculosis disease, in contrast to typical tuberculosis evolution from small nodules into branching opacities, lobular consolidation and cavity. PTB manifesting with CMNs usually occurs in young people and usually without the typical symptoms of tuberculotic wasting or respiratory symptoms at the early stage $(6,7)$. This type of disease generally has a good outcome after anti-tuberculosis treatment (6-8).

CMNs and galaxy sign are common in sarcoidosis (9). РTB with $\mathrm{CMNs}$ should be differentiated mainly from sarcoidosis. The diagnosis differential points include: (I) Compared with tuberculosis, sarcoidosis is more likely to have mediastinal and hilar lymphadenopathy; (II) tuberculotic CMNs are more likely to merge, and caseous changes are more likely to occur; (III) CMNs of PTB can be accompanied by tree-in-bud signs, while the CMNs around sarcoidosis lack the characteristics of centrilobular nodules of PTB.

\section{Reversed halo sign (RHS) manifestation of PTB}

RHS manifests as ground grass opacity (GGO) in the center surrounded by a continuous or discontinuous consolidation ring (Figure 5) (10). It has also been described as the "atoll sign" because of its resemblance to a coral atoll (11). Although initially thought to be specific for cryptogenic organizing pneumonia (OP) $(12,13)$, RHS has been described in many infectious diseases and noninfectious diseases such as pneumonia due to fungi, tuberculosis, sarcoidosis, pulmonary infarction, lung cancer and anti-neutrophil cytoplasm antibodies (ANCA)associated vasculitis $(10,11)$. Among them, the most common pathogens in infectious diseases are mucormycosis, aspergillus and tuberculosis $(10,14,15)$. Nevertheless, RHS is still a rare sign pattern in PTB, till now there is no exact data on its detection rate in PTB. Tuberculosis patients with RHS pattern tend to be relatively young; moreover, these patients commonly do not present with typical clinical symptoms nor complications of tuberculosis (16).

In PTB RHS, the wall of the "atoll island" is composed of nodular accumulation and fused into ring-band consolidation with coarse boundary, while the overall boundary is clear. RHS can be single or multiple, and mainly distribute in the upper and middle lung zones with non-segmental distribution (Figure 6). Similar to CMNs, multiple pulmonary RHS lesions may have similar appearance (Figure 6). The nodular appearance of the rings in the RHS, which is seen in patients with active tuberculous RHS and also reported in other granulomatous infectious processes including schistosomiasis and cryptococcosis, has been histologically proven to correlate to granulomas $(17,18)$. This helps the differentiation of active granulomatous disease $v s$. OP (19). 

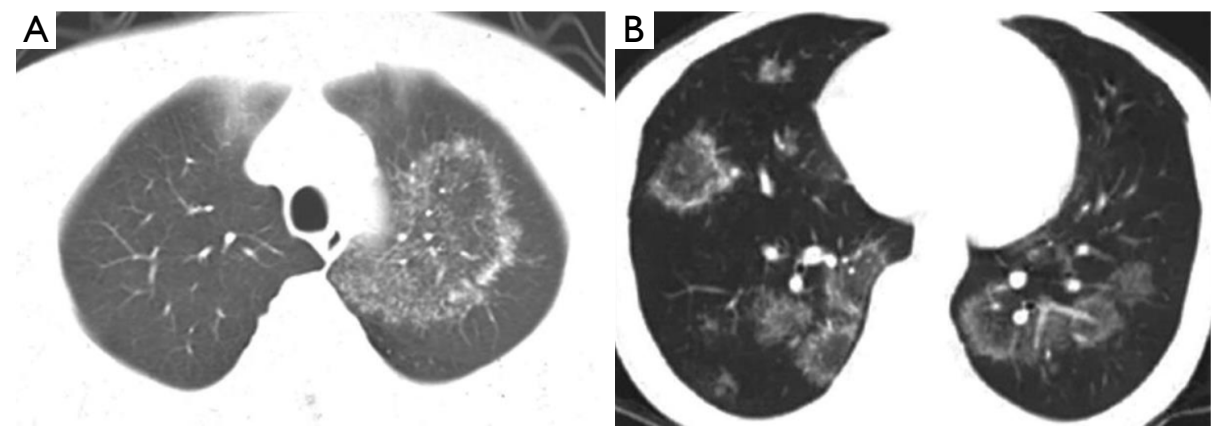

Figure 5 Computed tomography (CT) reversed halo sign (RHS) manifestation of pulmonary tuberculosis (PTB). (A) A 54-year-old male patient with PTB and negative sputum smear. He was asymptomatic and the PTB was noted during physical examination. CT shows a large single RHS in the upper left lobe distributed across lung segments. The ring wall is formed by the accumulation of micro-nodules with ground grass opacity and micro-nodules seen inside the ring. (B) A 25-year-old male patient with PTB and negative sputum smear. CT shows multiple RHS manifestations in both lungs, with high similarity in shape among the lesions.
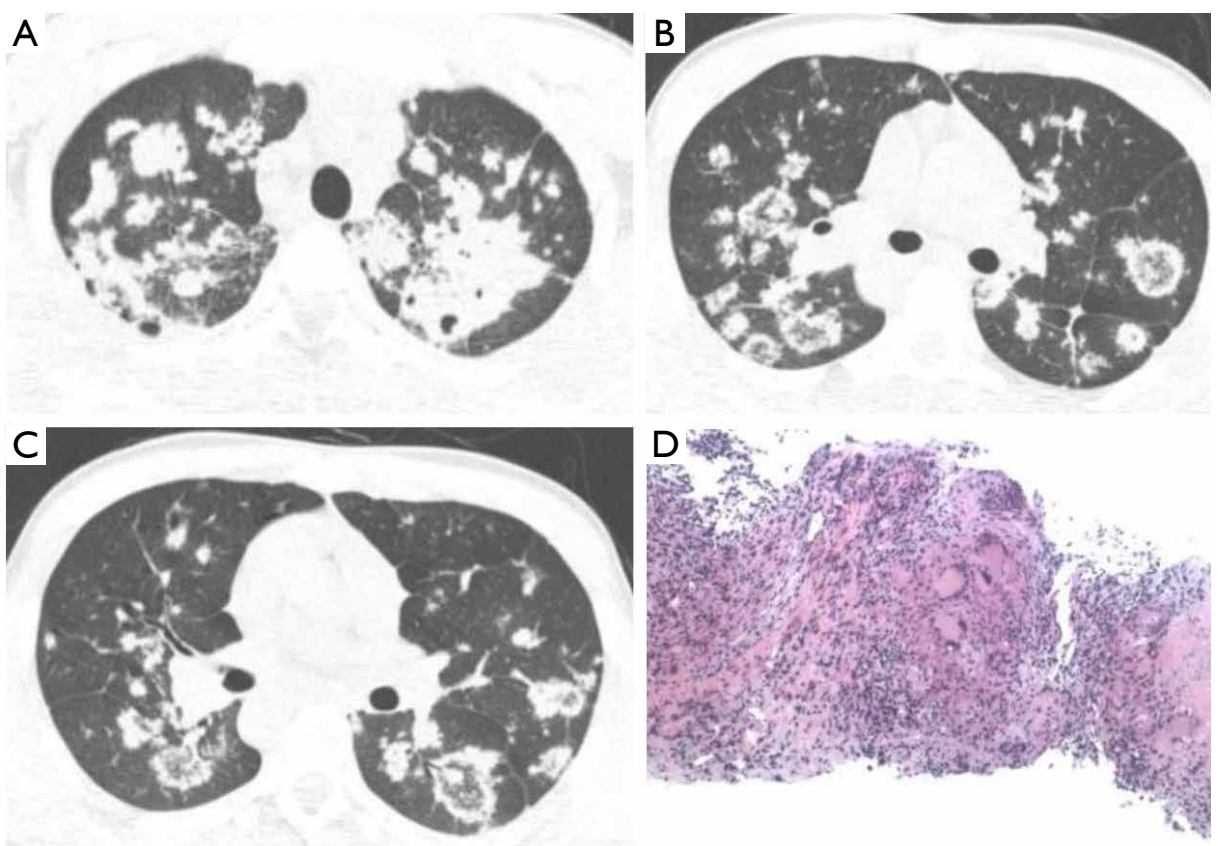

Figure 6 Computed tomography (CT) reversed halo sign (RHS) manifestation of pulmonary tuberculosis in a 26-year-old male pulmonary tuberculosis patient with right sided heart. (A,B,C): Multiple RHS in both lungs. The upper and middle regions of the lungs fused to form consolidation, and there are cavities in some consolidation areas. This case of RHS has appearance similar to CMNs. There are more lesions in upper lobes, and lesions have similar appearance. (D) CT-guided lung biopsy on the "ring" of RHS shows granulomas with caseous necrosis (HE stain, $\times 100)$.

In the process of disease progression, the RHS may undergo fusion and necrosis, forming lung consolidation or cavities, leading to the disappearance of RHS characteristics (Figure 6). After anti-tuberculosis treatment, the micro- nodules and GGO inside the RHS are absorbed firstly, followed by the thinning of the island wall and then its final absorption (Figure 7).

Our own observation suggests that CMNs and RHS are 

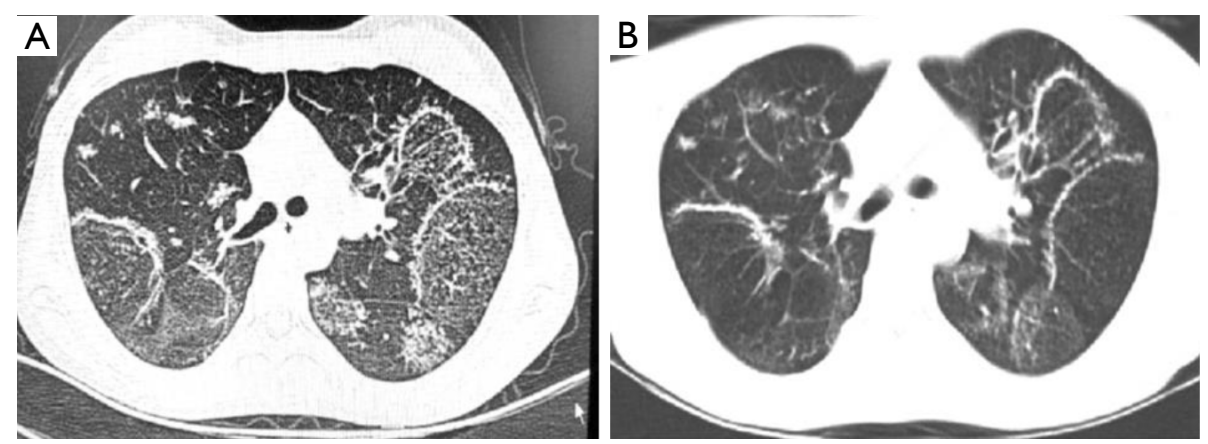

Figure 7 CT reversed halo sign (RHS) manifestation of pulmonary tuberculosis. An 18-year-old female patient with pulmonary tuberculosis involving the lungs, intestines, and meninges. (A) There are multiple RHS in both lungs, with clusters of micro-nodules and ground grass opacities distributed inside. (B) After anti-tuberculosis treatment, micro-nodules and ground grass opacities inside the wall were firstly absorbed. CT, computed tomography.
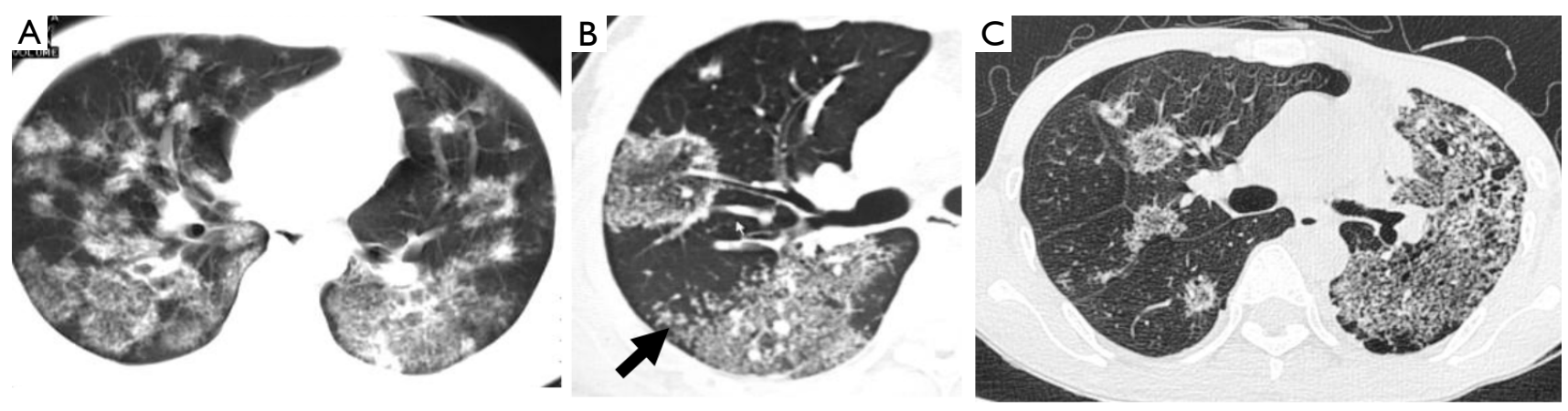

Figure 8 Pulmonary tuberculosis cases with co-existence of clustered micronodules (CMNs) and reversed halo sign (RHS). (A) A 26-yearold male tuberculosis patient. Multiple CMNs are seen in both lungs with a round-shape and faint consolidation ring visible in the periphery showing RHS resemblance. (B) A 36-year-old female tuberculosis patient with positive sputum smear. There are two lesions in the anterior and posterior parts of the right upper lobe. The anterior lesion shows a RHS pattern, and the posterior lesion shows a CMNs pattern. Tree-in-bud sign (black arrow) can be seen on the edge of the lesion. (C) A 58-year-old male multiple drug resistant (MDR) tuberculosis patient with positive sputum smear. CT shows multiple RHS in the right lung and CMNs in the upper lobe of the left lung. CT, computed tomography.

closely related. In some cases with CMNs, there can be a consolidation zone in the periphery and this demonstrates RHS-like performance, suggesting that CMNs lesion can induce the formation of RHS. Cases of CMNs coexist with RHS are also observed (Figure 8). It is possible that some CMNs manifestation can turn into the RHS manifestation.

Tuberculous RHS needs to be differentiated from various diseases that manifest signs similar to RHS. Tuberculous RHS has nodules on the wall and inside the wall, which allow rule out non-granulomatous RHS. In other granulomatous RHS, sarcoidosis RHS may also show nodular features, but it is often accompanied by bilateral symmetric hilar and mediastinal lymphadenopathy. Other granulomatous diseases, such as fungus and ANCA-related vasculitis, are not difficult to identify by their typical clinical features $(10,20)$. It should be noted that some tuberculosis related RHS can also manifest as non-nodular RHS. The existence of tree-in-bud accompanying RHS suggests the diagnosis of PTB.

\section{Pneumatocele manifestation of PTB}

Pulmonary pneumatoceles are thin-walled air-containing cavities in the lungs. They are usually secondary changes 

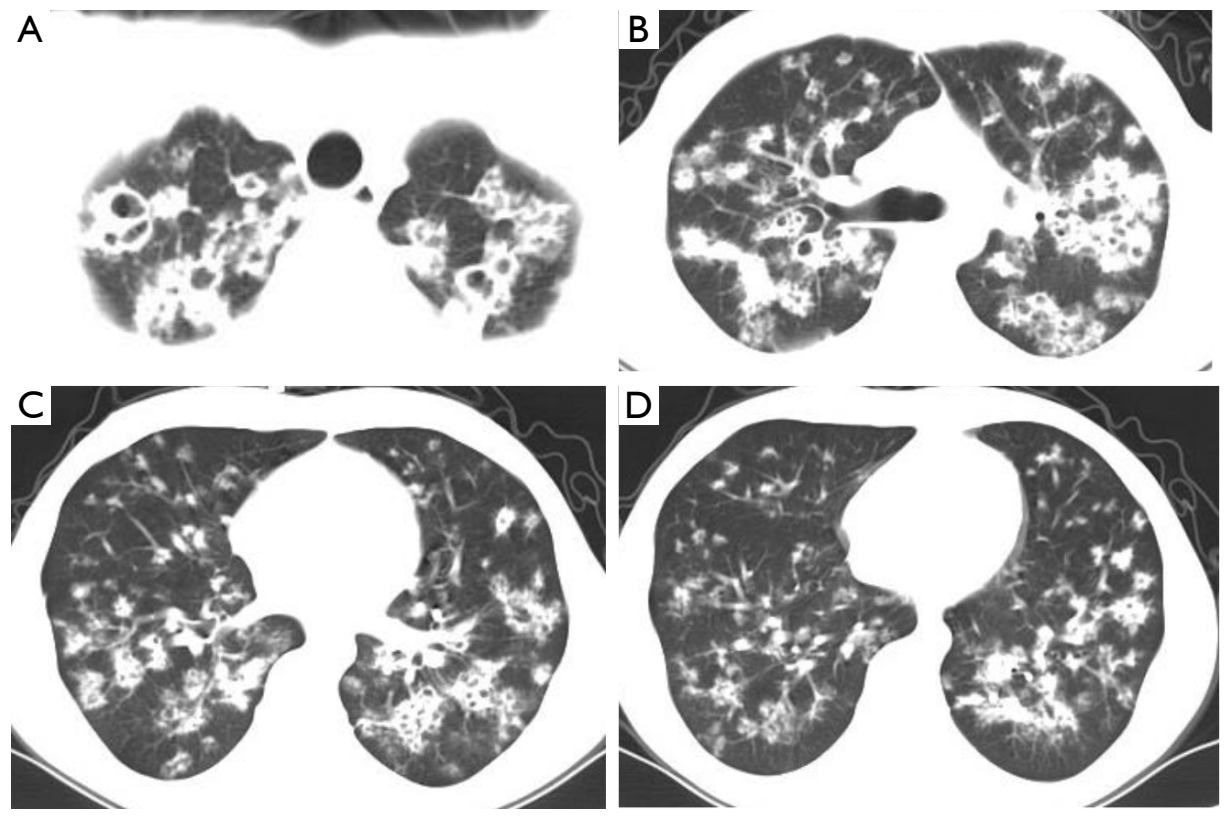

Figure 9 A 26-year-old male patient with pulmonary tuberculosis. Lung CT shows diffusely distributed nodules and cystic changes in both lungs, and the lower lung air sacs are small and distributed in clusters. Thickening of the airway wall can be seen in the diseased area. A bronchoscopy lung biopsy revealed chronic inflammation and a small extent of necrosis of the bronchial mucosal tissue, and positive acidfast staining. CT, computed tomography.

caused by acute infections. Pulmonary pneumatoceles can also be caused by inhalation of hydrocarbon fluids and trauma. The most common infectious pathogens for cystic disease are Staphylococcus aureus and Pneumocystis jiroveci pneumonia (PJP). Others infectious pathogens such as Streptococcus pneumoniae, Escherichia coli, and Bacillus mirabilis have also been reported to cause pulmonary pneumatoceles (21-26). Pulmonary pneumatoceles caused by tuberculosis are relatively rare (27-30). Ko et al. (29) speculated a number of modes of pathogenesis of the cystic lesions in tuberculous lung. Drainage of necrotic lung parenchyma in the areas of consolidation, coupled with check-valve bronchiolar obstruction caused by edematous luminal narrowing with mural inflammation of the involved bronchiole, may cause cyst formation. The cystic lesions may represent areas of dilated bronchioles, as do the cystic lesions in Langerhans cell histiocytosis (31).

Granuloma and caseous necrosis of the terminal bronchioles and respiratory bronchus, leading to stenosis of the lumen, causing a valve mechanism including cyst formation. Other possibilities included diffuse alveolar damage (DAD)-induced alveolar interstitial damage and drugs (isoniazid) caused lung air sacs.
The CT signs are usually diagnostic for tuberculous pneumatoceles, which may involve single or multiple lung lobes, or may diffusely distribute (Figure 9). Tuberculous cystic lesions are associated with surrounding areas of centrilobular lesions and cavitating nodules. Over the course, the lesions can increase in size and have a tendency to coalesce (Figure 10), or even rupture.

After anti-tuberculosis treatment, pneumatocele can be reversible (Figure 11) (29). However, if the interstitium is severely broken or necrotic, the residual air sacs may be reduced after treatment, but the cystic changes may remain as residual anomaly.

\section{Hematogenously disseminated PTB with predominantly diffuse GGO manifestation}

According to the presence or absence of miliary nodules, the imaging appearance of blood-borne tuberculosis with diffuse GGO can be divided into predominantly diffuse GGO manifestation and diffuse GGO with miliary nodules manifestation. The predominantly diffuse GGO manifestation of PTB mainly occurs in cases of hematogenously disseminated tuberculosis with DAD, 

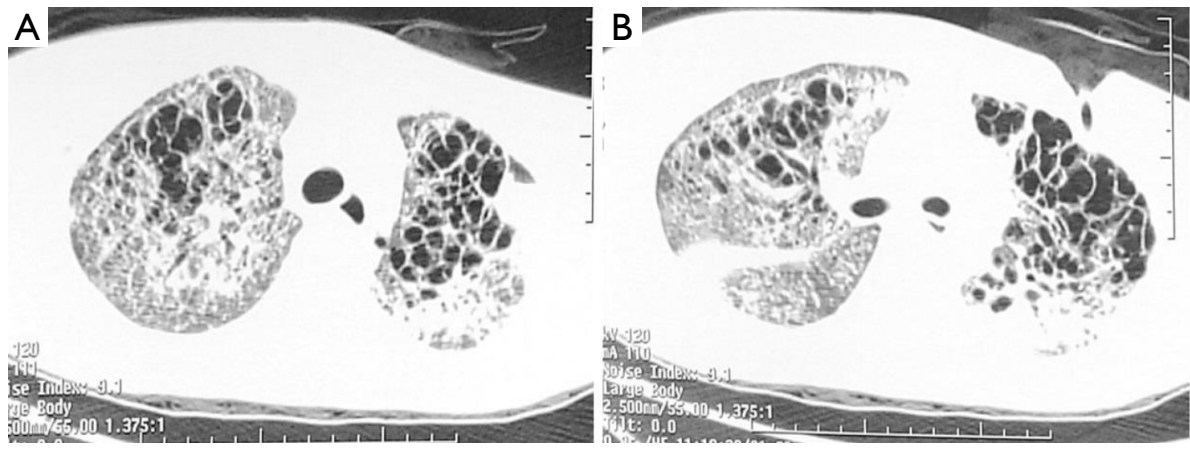

Figure 10 A 40-year-old male tuberculosis patient. Pathogen smear was positive during the initial diagnosis. There are multiple clusters of pneumatocele shown in the upper lobes of the lungs, with septal breaks in the larger cysts. GGO and multiple nodules are scattered around. GGO, ground grass opacity.
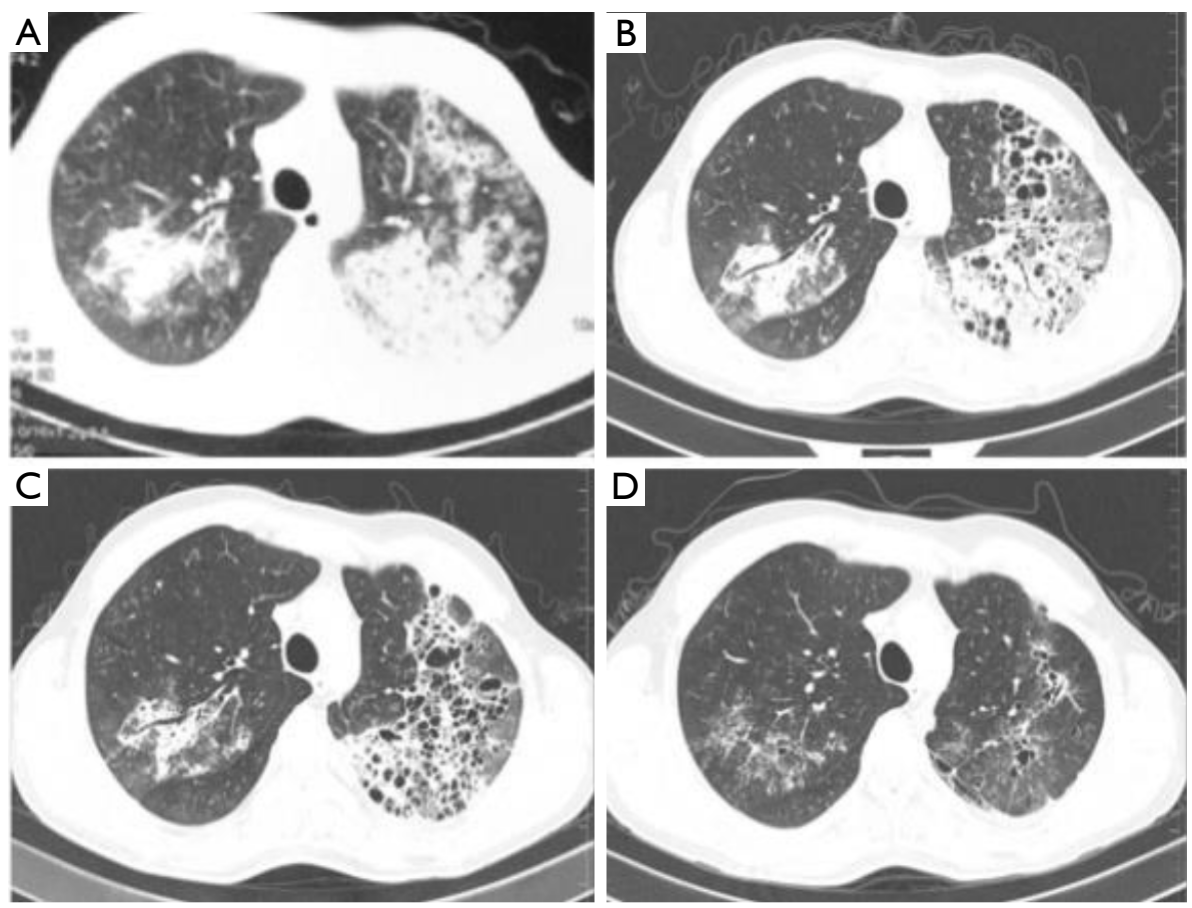

Figure 11 A 46-year-old male tuberculosis patient. Pathogen smear was positive during the initial diagnosis. Confluent lung consolidations with local honeycomb changes (A). After anti-tuberculosis treatment, consolidation was gradually replaced by pneumatoceles, and ground grass opacities gradually decreased (B: week-2 of treatment, C: week-4 of treatment). After 20 weeks of anti-tuberculosis treatment, most of the cystic degeneration area returned to normal, with nodules, fiber streaks and a small number of air sacs still remaining (D). 

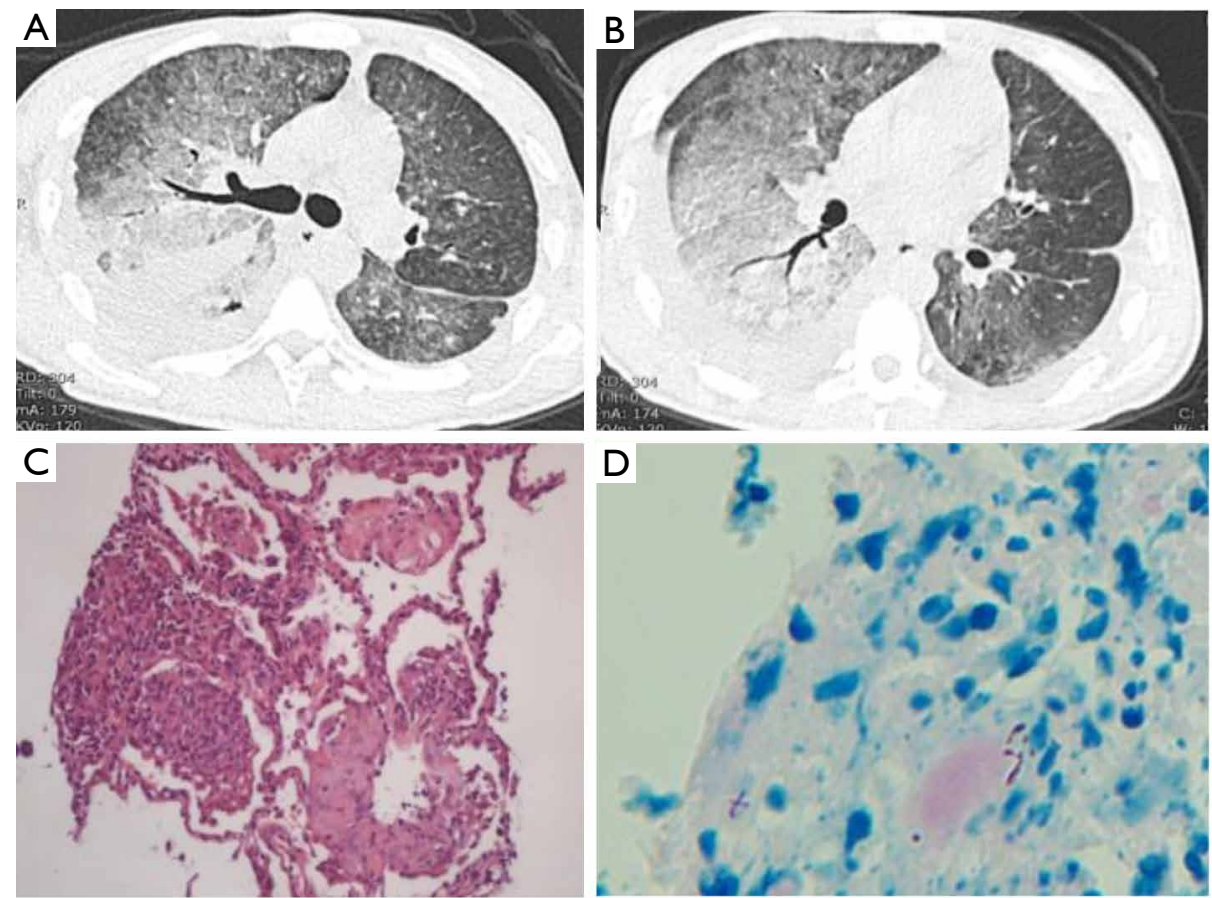

Figure 12 A 25-year-old male patient with blood-borne tuberculosis. (A,B) Diffuse asymmetrical distribution of GGO in both lungs, and local miliary micronodules. Lesions are more substantial in right lung and accompanied by right pleural effusion and consolidation of the right lower lung. (C) Percutaneous lung biopsy: focal epithelioid granulomatous lesions without apparent necrosis (HE stain $\times 100)$. (D) A sign of positive acid-fast staining in biopsied sample $(\times 400)$.

which is a high-risk factor for acute respiratory distress syndrome (ARDS) (32,33). Diffuse GGO mode is mostly due to impaired immune function, and granulomas do not form or form poorly (Figures 12,13). Blood-borne tuberculosis with predominantly diffuse GGO manifestation tends to progress rapidly and may be misdiagnosed initially. In secondary tuberculosis with this type of manifestation, GGO is often accompanied by pulmonary consolidation, cavities, nodules and other signs (32-36).

For the manifestation of diffuse GGO with miliary nodules, the existence of miliary nodules suggest blood-seeded tuberculosis. However, it is often difficult to identify miliary nodules in the GGO background, especially in the dense diffuse GGO background. In disseminated PTB dominated by GGO, these GGOs show a bilateral asymmetric distribution and the lower lungs may be more affected.

Due to the lack of typical signs, this type of PTB can be often misdiagnosed as viral pneumonia, PJP, and interstitial pneumonia, causing delay in diagnosis and management. Pathogenic examination for Mycobacterium tuberculosis should be pursued to obtain a definite diagnosis.

\section{Hematogenously disseminated PTB with randomly distributed non-miliary nodules}

Blood-borne tuberculosis with multiple nodules as the main feature can occur, though rare in clinical practice. Hematogenously disseminated PTB commonly manifest as diffusely distributed miliary nodules in both lungs and has the characteristics of distribution in similar size and similar density. Caseous necrosis can also occur. The imaging of this type of tuberculosis show multiple nodules and mass shadows in both lungs. Because the diameter of some large nodules exceeds the structure of secondary lung lobules, there are certain difficulties in judging the distribution characteristics of these nodules. A preliminary judgment of blood-borne spread can be made according to the subpleural distribution and the distribution pattern along blood vessel branches while without a tendency to follow segmental distribution (i.e., so called feeling vessel sign) (Figure 14). Note, in practice, lung tuberculosis of this type of findings is often misdiagnosed as malignant tumor lung metastasis $(37,38)$.

Hematogenously disseminated PTB may be accompanied 

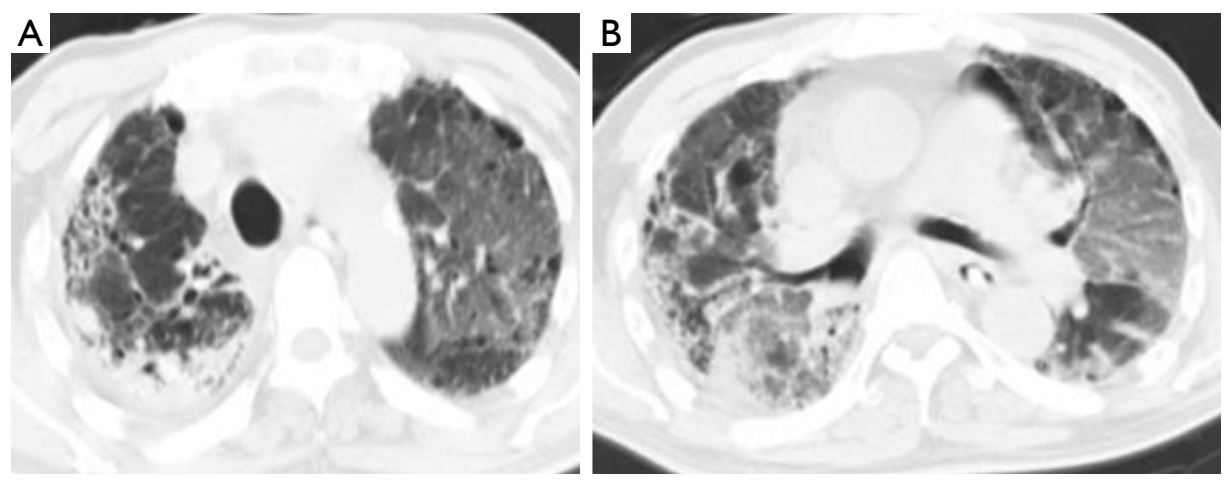

Figure 13 A female patient of 65 years old. She had myelodysplastic syndrome (MDS), blood-borne tuberculosis, and tuberculous meningitis. The sputum tuberculosis smear was positive. Chest CT shows GGOs diffusely distribute in both lungs, subpleural lung consolidation in the right lung, and no apparent nodule seen. The background of emphysema shows pneumatocele changes against the backdrop of GGO. The patient eventually died of acute respiratory distress syndrome and multiple organ failure. GGO, ground glass opacity; CT, computed tomography.

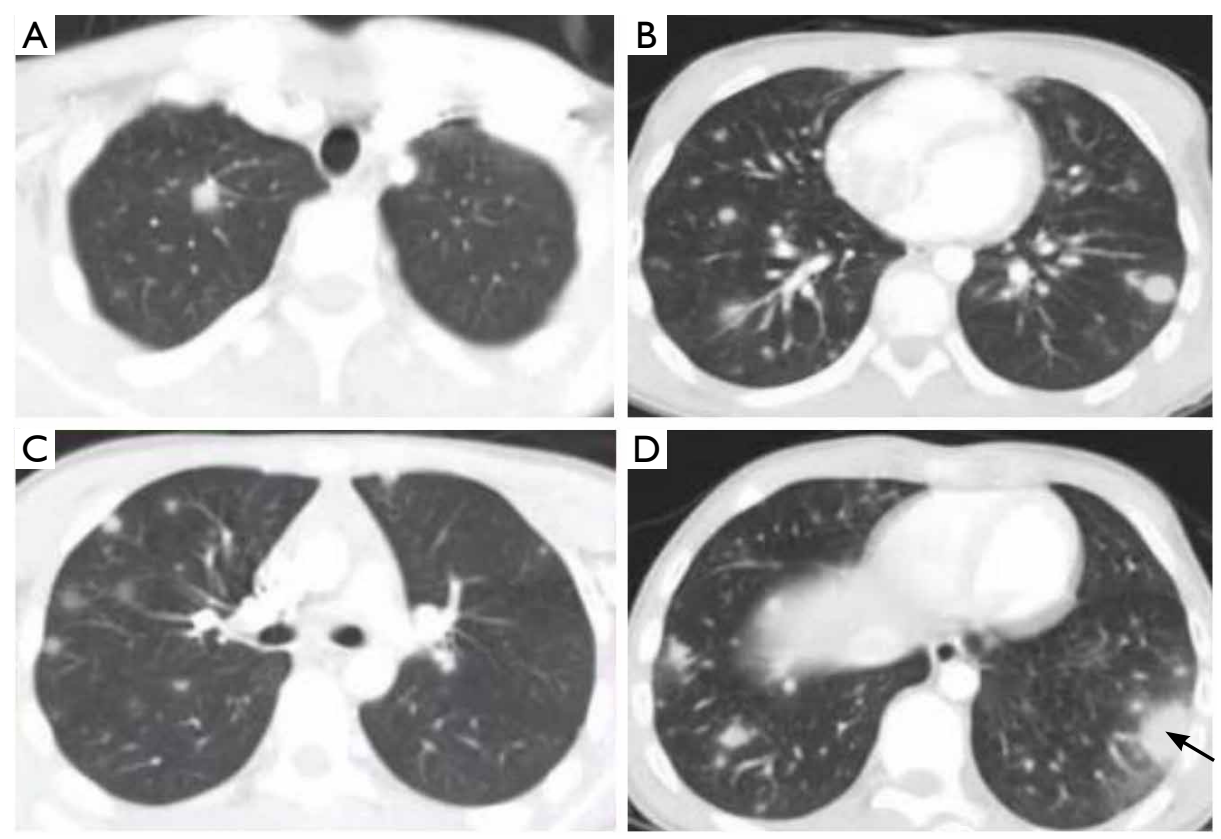

Figure 14 A female patient of 21 years old with high fever for 2 weeks. Lung CT shows multiple nodules of different sizes in both lungs, with the distribution pattern along blood vessel branches and being non-segmental (consistent with feeling vessel sign). A lung biopsy (black arrow) revealed a granuloma with caseous necrosis and a positive acid-fast stain. Diagnosis: hematogenously disseminated tuberculosis. CT, computed tomography.

by pleural effusion and multiple organ involvement can be seen. Positron emission tomography (PET)/CT examination has an important value in the assessment of blood-borne spreading $(39,40)$. By discovering the high ${ }^{18} \mathrm{~F}-\mathrm{FDG}$ uptake of tuberculosis lesions in multiple tissues and organs, combined with multiple nodules that are randomly distributed in the lung (without segmental distribution pattern), it can help to suggest blood-borne seeding 

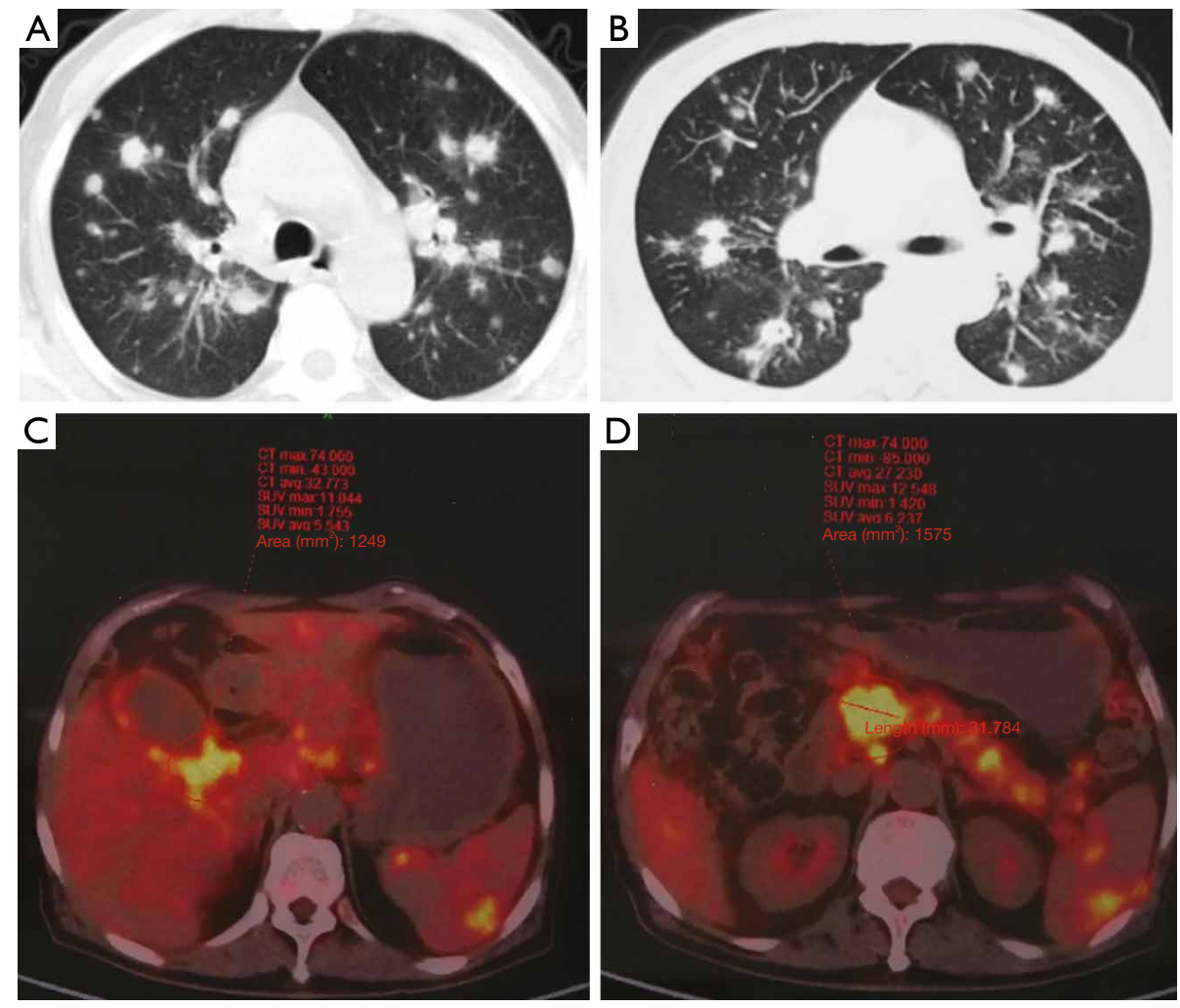

Figure 15 A 73 -year-old male patient with tuberculosis. The initial sputum spear was negative. (A,B) Multiple symmetrical and random (non-segmental) distribution of nodular lesions of varying sizes in both lungs. (C,D) PET/CT: nodular increased radioactivity in the liver, pancreas, and spleen. Percutaneous lung biopsy: coagulation necrosis with granulomatous lesions, acid-fast staining (+), sputum Mycobacterium tuberculosis DNA: $4.9 \times 10^{3}$ copies/mL. PET, positron emission tomography; CT, computed tomography.

\section{(Figure 15).}

This type of PTB usually represent the subacute or chronic stage of tuberculosis evolution processes. Differential diagnosis mainly includes tumor metastasis, septic pulmonary embolism, granulomatous polyangiitis. The metastatic multiple nodules do not have other associated PTB signs. Lung metastases of malignant tumors tend to have relatively clear borders, while without fibrous cords or spiculated nodules. On the other hand, the nodules of tuberculosis may develop caseous necrosis and fiber encapsulation over time. After contrast agent injection, ring enhancement of contrast can be noted in some tuberculous nodules. Septic pulmonary embolism has a rapid onset and often have apparent symptoms of infection, with the peripheral white blood cells and procalcitonin significantly increased, which help to differentiate. Granulomatous vasculitis has the typical triad of sinusitis, renal function damage and pulmonary necrotizing nodules.

\section{PTB changes occur on the background of emphysema or honeycomb changes of interstitial pneumonia}

Tuberculosis lesions in the emphysema area and the honeycomb-like change areas of interstitial pneumonia often have unique imaging features. PTB changes occurred on the background of emphysema or honeycomb-like changes of interstitial pneumonia can be confused with the lung cavities of tuberculosis. The former occurs on the basis of structural damage of emphysema or honeycomblike change due to interstitial pneumonia. The latter is directly caused by tuberculosis. During the process of pathology progression, the tuberculous consolidation may spread along the interstitium of the emphysema and lose its characteristics of segmental distribution. Tuberculosis occurs in emphysema area can manifest as non-segmental consolidation and GGO, similar to pneumonia (41-43). Due to the idle air cavity caused by the emphysema area, 


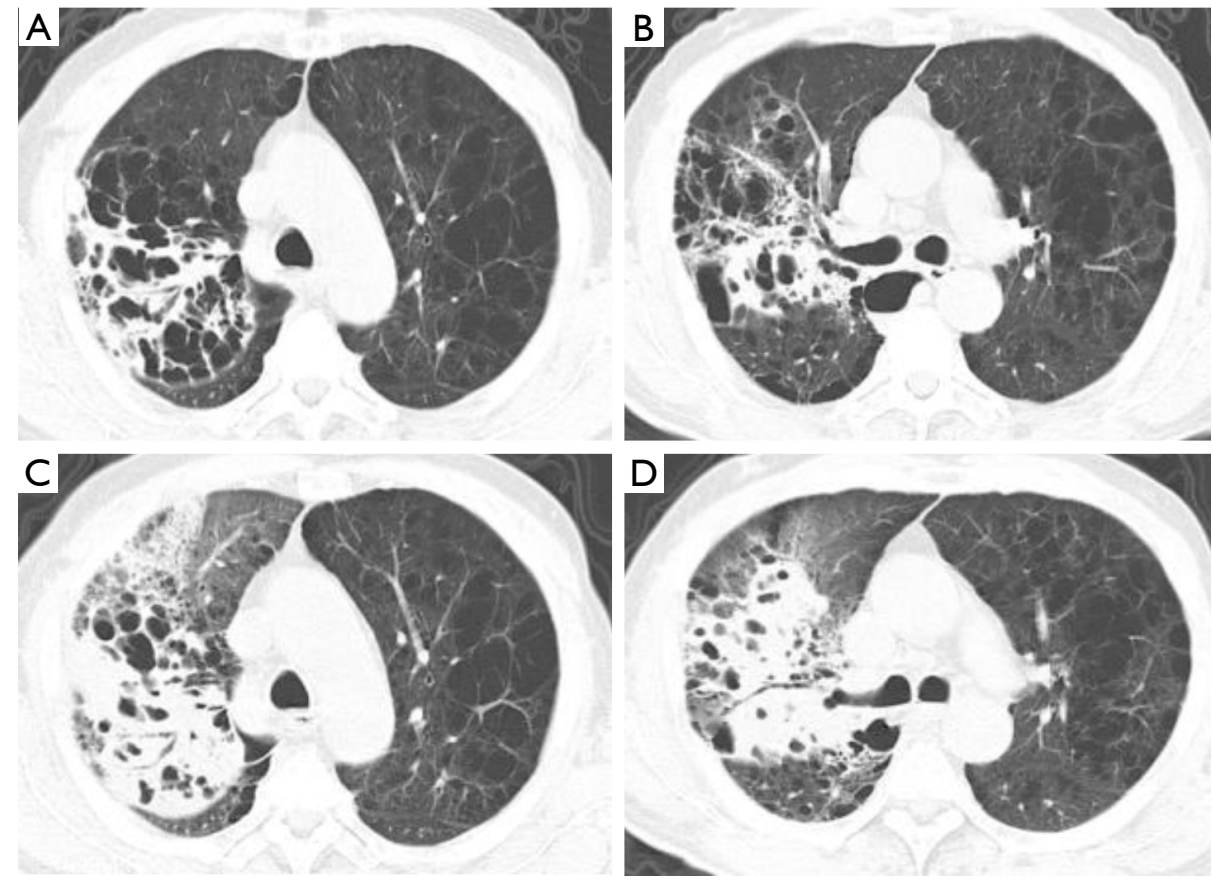

Figure 16 A 68-year-old female emphysema patient with tuberculosis and positive sputum smear. (A,B) Computed tomography (CT) shows pan-lobular emphysema and consolidation of the right lung with multiple air-containing cavities. (C,D) In the same case re-examination 9 days later, CT shows the lesions spread along the emphysema area with pseudo-cavity.

the cyst wall of emphysema can be observed in the lung consolidation and GGO area, which is prone to be mistaken as the "worm-like" cavity of caseous pneumonia. A case of PTB occur on the background of emphysema is shown by Figure 16.

Figure 17 shows that a case of tuberculosis consolidation involved the subpleural emphysema area, which appears as a lamellar "honeycomb" or "polycystic" consolidation in the dorsal area. The distribution characteristics of tuberculosis is mainly related to expansion of the alveoli in the emphysema area, the expansion of alveolar holes, and rupture of the alveolar interval. The Mycobacterium tuberculosis is more likely to spread to the entire emphysematous region. Tuberculosis lesions in the emphysema area lack central lobular nodules or tree bud signs (41-43), which is a potential cause for misdiagnosis (e.g., misdiagnosed as other infectious diseases). This is related to the destruction of the lobular central structure of emphysema and lack of anatomy basis for the formation of tree bud signs.

In patients with chronic interstitial lung disease, due to the use of steroids and/or immunosuppressants and the honeycomb-like structural destruction of lung tissue and emphysema, the patients have increased susceptibility to Mycobacterium tuberculosis because of the decreased clearing effect in the damaged lung or the easier attachment of bacilli to the destroyed alveolar surface $(44,45)$. Because interstitial fibrosis and honeycombed lungs mainly occur in the lower lobes, distribution of tuberculosis in lower lobes of lung also increases. Tuberculous changes with interstitial pneumonia tend to dominate by pulmonary consolidation $(46,47)$ (Figure 18). The typical features of post-primary tuberculosis such as centrilobular nodules or tree-inbud lesions are infrequently found in these patients (46). Atypical manifestation of PTB is common in patients with idiopathic pulmonary fibrosis (IPF), which may mimic lung cancer or bacterial pneumonia (46). 


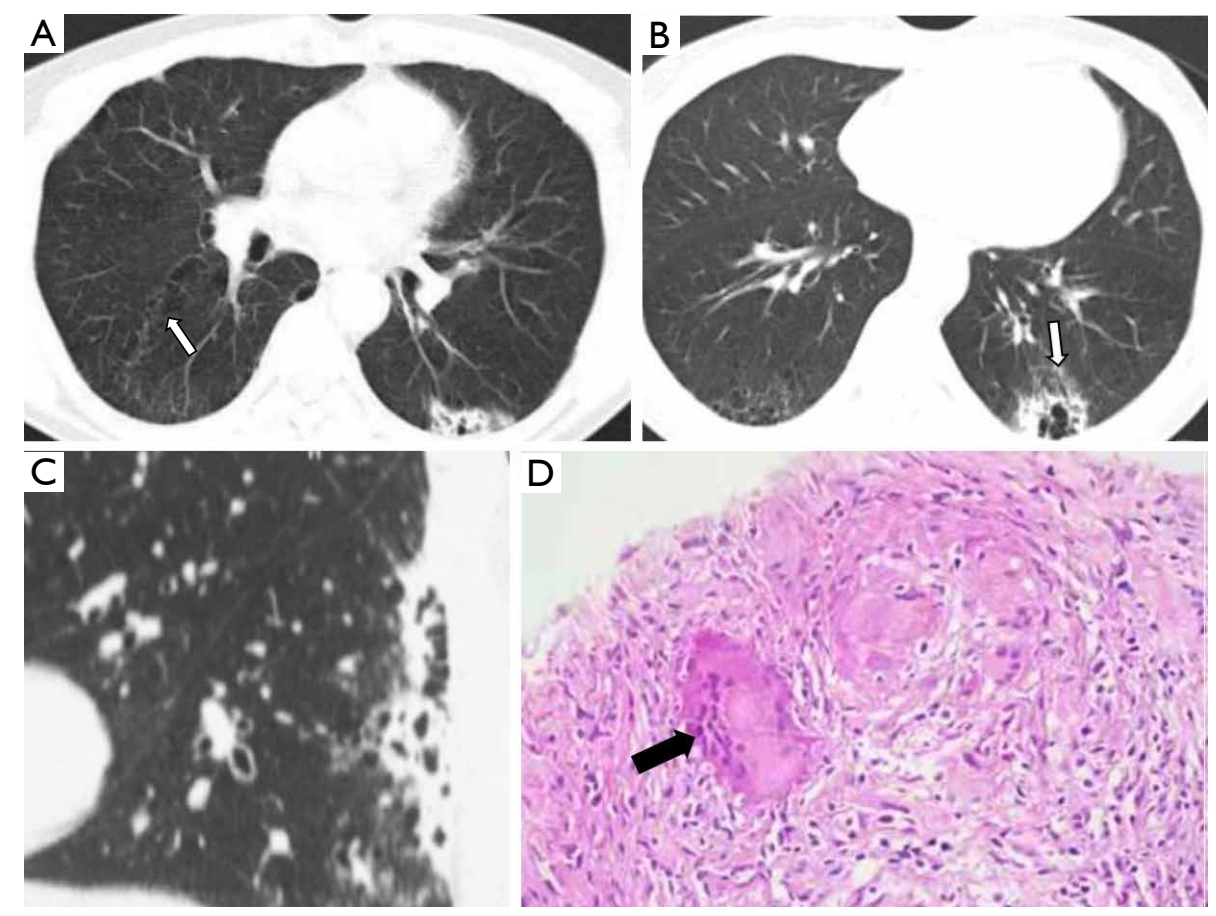

Figure 17 A 56-year-old male tuberculosis patient with positive sputum smear. CT showed (A,B) consolidation in the emphysematous area of the lower lobe of the left lung and clustered air sacs appearance. The emphysematous change at the contralateral side (the dorsal side of the right lower lobe) can serve as a control. The re-constructed sagittal plane (C) shows the lesions extend along the pleural surface with a non-segmental distribution. (D) Histology: inflammatory consolidation combined with non-necrotizing granulomatous inflammation, accompanied by multinucleated giant cells (black arrow; HE stain, 200× magnification). PCR positive for Mycobacterium tuberculosis (courtesy of Professor Chuan-Shu Sun, Department of Radiology, Dalian Medical University, China). CT, computed tomography.

\section{PTB manifesting as OP}

OP is a pulmonary inflammatory process characterized by the presence of granulation tissue that fills the distal bronchioles, respiratory bronchioles, bronchiolar ducts, and alveoli (48-50). Secondary OP refers to OP with a clear cause. Common pathogenic factors include infection (such as bacteria, viruses, mycoplasma), connective tissue diseases, drugs (such as amiodarone, bleomycin), radiotherapy, organ transplantation. In rare cases, Mycobacterium tuberculosis has been reported as a causative factor for OP (51-53).

The mechanism of tuberculous OP development is not fully understood. The imaging manifestations of tuberculous $\mathrm{OP}$ are not specific. Tuberculous OP often appears as focal pulmonary consolidation and GGO, while without the typical tuberculosis signs of tree-in-bud, caseous necrosis, and cavities (49-51) (Figures 19,20). According to limited literature $(52,53)$ and authors' own experience, tuberculous OP more likely to have negative sputum biology. Molecular pathology techniques such as PCR amplification of biopsy tissue can be used to improve the pathogen detection rate $(52,53)$. If Mycobacterium tuberculosis infection is identified early and anti-tuberculosis intervention given at early stage, the OP process is reversible (Figure 20).

In conclusion, PTB may manifest a number of atypical CT imaging changes. While the overall incidence of PTB is decreasing, the incidence of atypical manifestations of tuberculosis is increasing. This increases the possibility of misdiagnosis for PTB. A good understanding of these atypical imaging changes of active PTB shall help the diagnosis and differential diagnosis in clinical practice. 

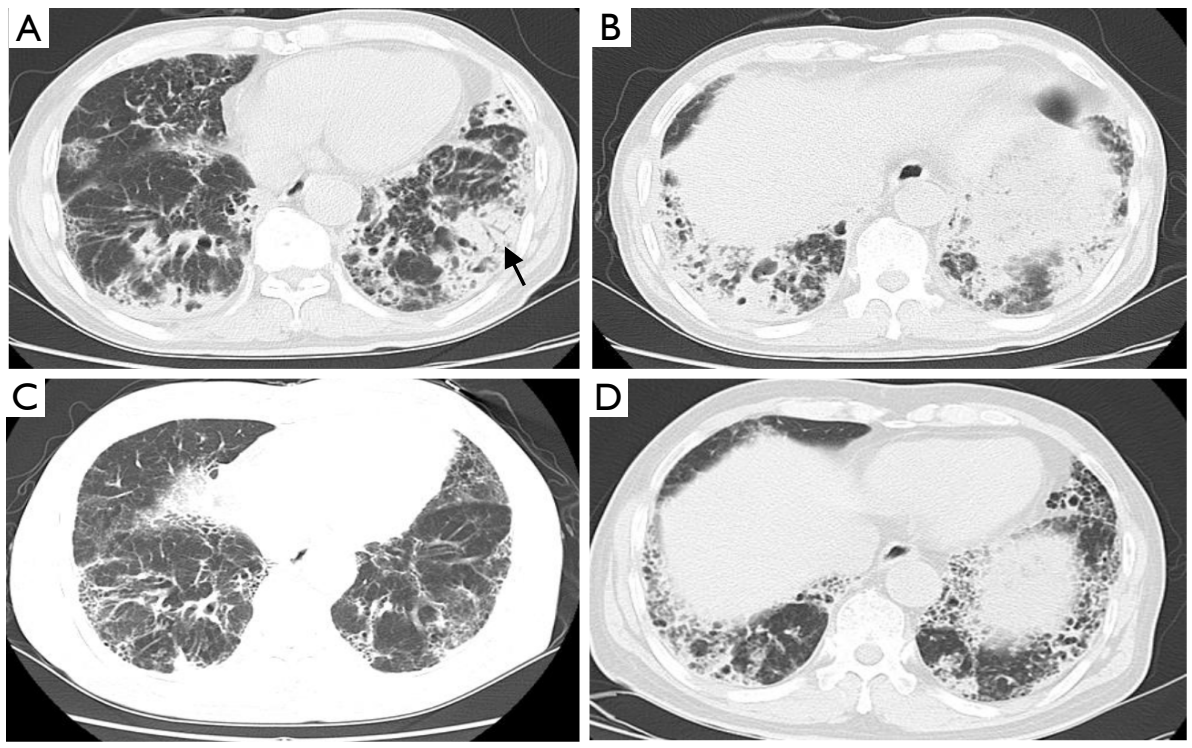

Figure 18 A 67-year-old male patient with idiopathic pulmonary fibrosis (IPF). Right lower lobe bronchoalveolar lavage fluid (BALF) tested positive for Mycobacterium tuberculosis. Computed tomography (CT) shows irregular consolidation in the interstitial fibrosis area (black arrow) (A,B). One year after anti-tuberculosis treatment (C,D), the lung consolidations were largely absorbed, revealing the previously existing cellular changes near the pleura of both lungs, in line with IPF.
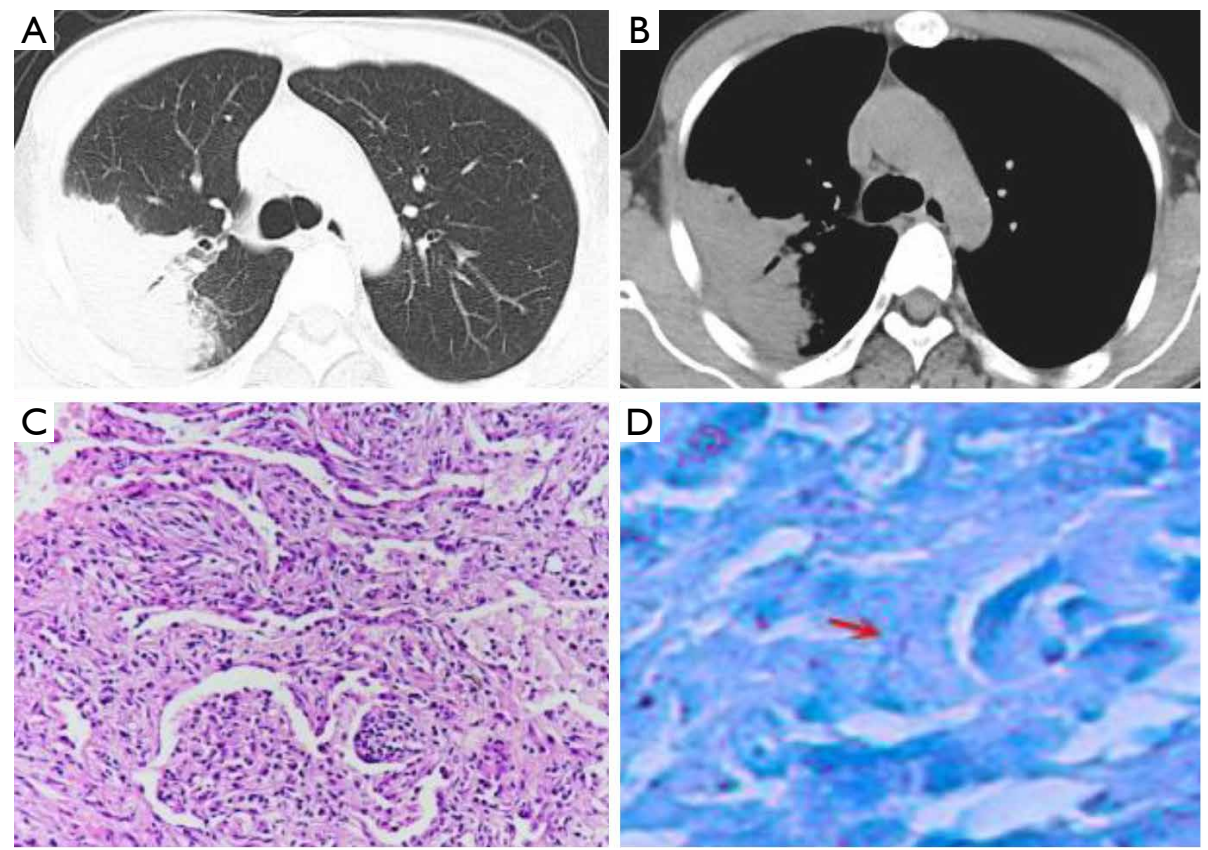

Figure 19 A 39-year-old male tuberculosis patient with negative sputum test. (A,B) Chest computed tomography (CT): large consolidation and GGO of the right lung, with signs of air bronchogram, no obvious low-density necrosis area is noted. (C) CT-guided percutaneous lung biopsy: lung interstitial fibrous tissue hyperplasia with lymphocyte and plasma cell infiltration in the upper lobe of the right lung, loose emboli of granulation tissue seen in the alveoli and respiratory bronchioles (HE stain, $\times 400$ ), in line with OP characteristics. (D) Acid-fast staining $(\times 400)$ of the tissue specimen note Mycobacterium tuberculosis (red arrow). GGO, ground glass opacity. 


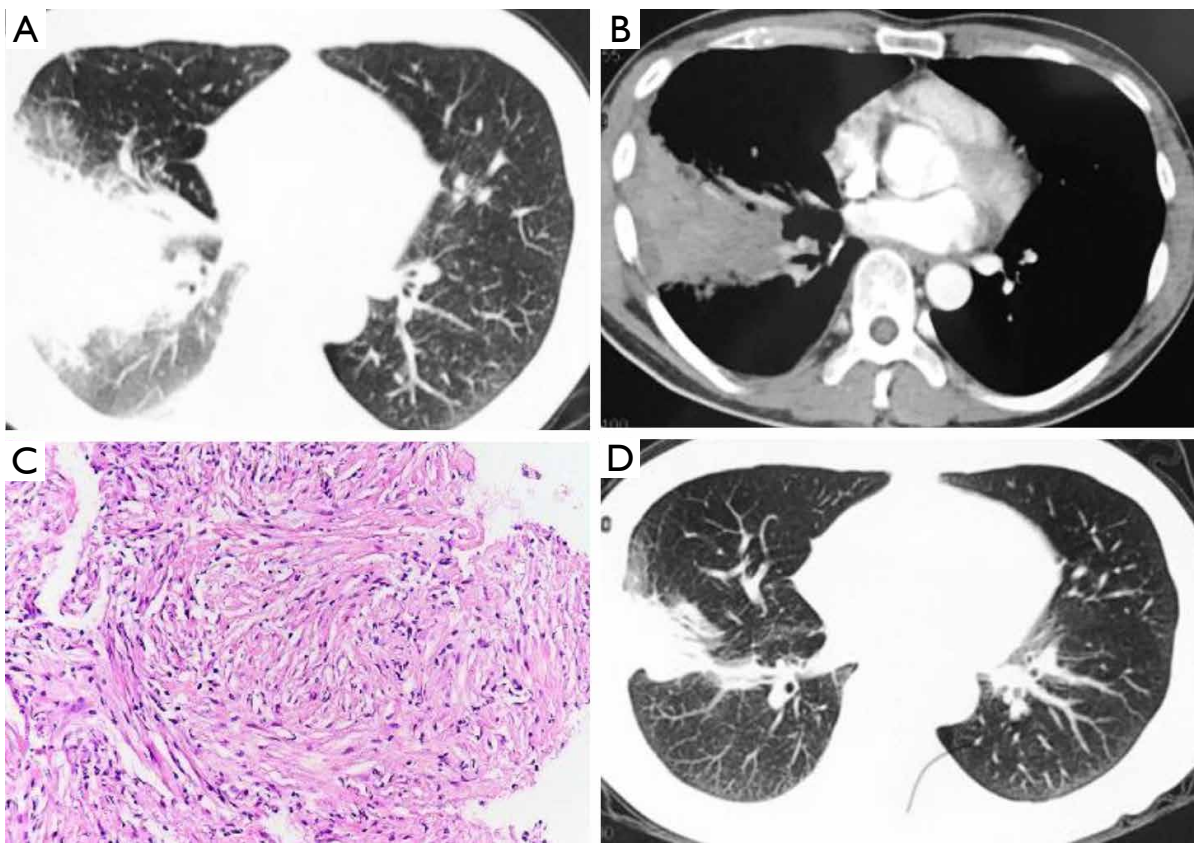

Figure 20 A 44-year-old male patient with tuberculous organizing pneumonia. The sputum test was negative for Mycobacterium tuberculosis. (A) Computed tomography (CT) shows a large area of consolidation in the right lung with ground glass opacites around the edge of the lesion. (B) Contrast enhanced CT shows uneven enhancement without cavity. (C) CT-guided percutaneous lung biopsy: bronchiolitis, tissue cell deposition in the alveolar cavity, interstitial fibrous tissue hyperplasia. Histopathological consideration: organizing pneumonia (HE stain, $\times 400$ ). (D) Re-examination of chest CT after 45 days of anti-tuberculosis treatment, the lesion was substantially absorbed.

\section{Acknowledgments}

Funding: None.

\section{Footnote}

Conflicts of Interest: All authors have completed the ICMJE uniform disclosure form (available at http://dx.doi. org/10.21037/qims-20-1323). YXJW serves as an Editor-inChief of Quantitative Imaging in Medicine and Surgery. The authors have no other conflicts of interest to declare.

Open Access Statement: This is an Open Access article distributed in accordance with the Creative Commons Attribution-NonCommercial-NoDerivs 4.0 International License (CC BY-NC-ND 4.0), which permits the noncommercial replication and distribution of the article with the strict proviso that no changes or edits are made and the original work is properly cited (including links to both the formal publication through the relevant DOI and the license). See: https://creativecommons.org/licenses/by-nc-nd/4.0/.

\section{References}

1. World Health Organization. Global tuberculosis report 2018. Available online: www.who.int/tb/publications/ global_report (accessed 15 March 2019).

2. Kobashi Y, Mouri K, Fukuda M, Yoshida K, Miyashita N, Oka M. Transitional change in the clinical features of pulmonary tuberculosis. Respiration 2008;75:304-9.

3. Kobashi Y, Mouri K, Yagi S, Obase Y, Miyashita N, Okimoto N, Matsushima T, Oka M. Clinical features of immunocompromised and nonimmunocompromised patients with pulmonary tuberculosis. J Infect Chemother 2007;13:405-10.

4. Wáng YX, Chung MJ, Skrahin A, Rosenthal A, Gabrielian A, Tartakovsky M. Radiological signs associated with pulmonary multi-drug resistant tuberculosis: an analysis of published evidences. Quant Imaging Med Surg 2018;8:161-73.

5. Geng E, Kreiswirth B, Burzynski J, Schluger NW. Clinical and radiographic correlates of primary and reactivation tuberculosis: a molecular epidemiology study. JAMA 
2005;293:2740-5.

6. Hong JH, Yoon SH, Goo JM, Yim JJ, Jeon YK. Clustered micronodules as predominant manifestation on CT: A sign of active but indolently evolving pulmonary tuberculosis. PLoS One 2020;15:e0231537.

7. Heo JN, Choi YW, Jeon SC, Park CK. Pulmonary tuberculosis: another disease showing clusters of small nodules. AJR Am J Roentgenol 2005;184:639-42.

8. Ko JM, Park HJ, Kim CH. Clinicoradiologic evidence of pulmonary lymphatic spread in adult patients with tuberculosis. AJR Am J Roentgenol 2015;204:38-43.

9. Nakatsu M, Hatabu H, Morikawa K, Uematsu H, Ohno Y, Nishimura K, Nagai S, Izumi T, Konishi J, Itoh H. Large coalescent parenchymal nodules in pulmonary sarcoidosis: "sarcoid galaxy" sign. AJR Am J Roentgenol 2002;178:1389-93.

10. Godoy MC, Viswanathan C, Marchiori E, Truong MT, Benveniste MF, Rossi S, Marom EM. The reversed halo sign: update and differential diagnosis. Br J Radiol 2012;85:1226-35.

11. Zompatori M, Poletti V, Battista G, Diegoli M. Bronchiolitis obliterans with organizing pneumonia (BOOP), presenting as a ring-shaped opacity at HRCT (the atoll sign). A case report. Radiol Med 1999;97:308-10

12. Voloudaki AE, Bouros DE, Froudarakis ME, Datseris GE, Apostolaki EG, Gourtsoyiannis NC. Crescentic and ringshaped opacities. CT features in two cases of bronchiolitis obliterans organizing pneumonia (BOOP). Acta Radiol 1996;37:889-92.

13. Kim SJ, Lee KS, Ryu YH, Yoon YC, Choe KO, Kim TS, Sung KJ. Reversed halo sign on high-resolution CT of cryptogenic organizing pneumonia: diagnostic implications. AJR Am J Roentgenol 2003;180:1251-4.

14. Maturu VN, Agarwal R. Reversed halo sign: a systematic review. Respir Care 2014;59:1440-9.

15. Zhan X, Zhang L, Wang Z, Jin M, Liu M, Tong Z. Reversed Halo Sign: Presents in Different Pulmonary Diseases. PLoS One 2015;10:e128153.

16. Zhan X, Wang Z, Zhang L, Jin ML, Liu M, Chen WH, Dai HP. Clinical and pathological features of adult pulmonary tuberculosis with reversed halo sign. Int $\mathrm{J}$ Tuberc Lung Dis 2013;17:1621-5.

17. Marchiori E, Grando RD, Simões Dos Santos CE, Maffazzioli Santos Balzan L, Zanetti G, Mano CM, Gutierrez RS. Pulmonary tuberculosis associated with the reversed halo sign on high-resolution CT. Br J Radiol 2010;83:e58-60.

18. Nattusamy L, Madan K, Bhalla AS, Guleria R. Reversed halo sign in active pulmonary tuberculosis. BMJ Case Rep 2014;2014:bcr2013202981.

19. Marchiori E, Zanetti G, Irion KL, Nobre LF, Hochhegger B, Mançano AD, Escuissato DL. Reversed halo sign in active pulmonary tuberculosis: criteria for differentiation from cryptogenic organizing pneumonia. AJR Am J Roentgenol 2011;197:1324-7.

20. Marchiori E, Zanetti G, Hochhegger B, Irion KL. Sarcoidosis and the reversed halo sign. Radiographics 2011;31:892-3.

21. Colling J, Allaouchiche B, Floccard B, Pilleul F, Monneuse O, Tissot E. Pneumatocele formation in adult Escherichia coli pneumonia revealed by pneumothorax. J Infect 2005;51:e109-11.

22. Ryu JH, Swensen SJ. Cystic and cavitary lung diseases: focal and diffuse. Mayo Clin Proc 2003;78:744-52.

23. Goodman PC. Pneumocystis carinii pneumonia. J Thorac Imaging 1991;6:16-21.

24. Lu PX, Deng YY, Liu ST, Liu Y, Liu YX, Wang YX, Zhu WK, Le XH, Yu WY, Zhou BP. Correlation between imaging features of Pneumocystis Jiroveci Pneumonitis (PCP), CD(4) (+) T lymphocyte count, and plasma HIV viral load: A study in 50 consecutive AIDS patients. Quant Imaging Med Surg 2012;2:124-9.

25. Boisset GF. Subpleural emphysema complicating staphylococcal and other pneumonias. J Pediatr 1972;81:259-66.

26. Meyers HI, Jacobson G. Staphylococcal pneumonia in children and adults. Radiology 1959;72:665-71.

27. Wan-Hsiu L, Sheng-Hsiang L, Tsu-Tuan W. Pneumatocele formation in adult pulmonary tuberculosis during antituberculous chemotherapy: a case report. Cases J 2009;2:8570.

28. Ray A, Suri JC, Sen MK, Khanna A. Cystic lung disease in tuberculosis: An unusual presentation. Lung India 2013;30:351-3.

29. Ko KS, Lee KS, Kim Y, Kim SJ, Kwon OJ, Kim JS. Reversible cystic disease associated with pulmonary tuberculosis: radiologic findings. Radiology 1997;204:165-9.

30. Takemura T, Akiyama O, Yanagawa T, Ikushima S, Ando T, Oritsu M. Pulmonary tuberculosis with unusual cystic change in an immunocompromised host. Pathol Int 2000;50:672-7.

31. Brauner MW, Grenier P, Mouelhi MM, Mompoint D, Lenoir S. Pulmonary histiocytosis X: evaluation with highresolution CT. Radiology 1989;172:255-8.

32. Lee J, Lim JK, Seo H, Lee SY, Choi KJ, Yoo SS, Lee SY, Cha SI, Park JY, Kim CH. Clinical relevance of ground 
glass opacity in 105 patients with miliary tuberculosis. Respir Med 2014;108:924-30.

33. Nakao A, Ishii H, Igata F, Kushima H, Fujita M, Hisano S, Watanabe K. Disseminated tuberculosis with acute respiratory distress syndrome lacking granuloma formation in the lung. J Infect Chemother 2016;22:638-41.

34. Fujita J, Bandoh S, Kubo A, Ishii T, Kanaji N, Nakamura H, Higa F, Tateyama M, Ishida T. HRCT shows variations in appearance in disseminated tuberculosis in adults. Int $\mathrm{J}$ Tuberc Lung Dis 2006;10:222-6.

35. Choi D, Lee KS, Suh GY, Kim TS, Kwon OJ, Rhee $\mathrm{CH}$, Han J. Pulmonary tuberculosis presenting as acute respiratory failure: radiologic findings. J Comput Assist Tomogr 1999;23:107-13.

36. Hashemian SM, Tabarsi P, Karam MB, Kahkouee S, Marjani M, Jamaati H, Shekarchi N, Mohajerani SA, Velayati AA. Radiologic manifestations of pulmonary tuberculosis in patients of intensive care units. Int $\mathrm{J}$ Mycobacteriol 2015;4:233-8.

37. Suzuki A, Hujita A, Yamamoto H, Ohtsuka T, Tokuda H, Suyama T. Pulmonary Tuberculosis Presenting Multiple Nodular Shadows in 4 Patients. Nihon Kokyuki Gakkai Zasshi 1999;37:538-42

38. Ariyürek MO, Karçaaltincaba M, Demirkazik FB, Akay H, Gedikoglu G, Emri S. Bilateral multiple pulmonary tuberculous nodules mimicking metastatic disease. Eur J Radiol 2002;44:33-6.

39. Hou S, Shen J, Tan J. Case report: multiple systemic disseminated tuberculosis mimicking lymphoma on 18F-FDG PET/CT. Medicine (Baltimore) 2017;96:e7248.

40. Yu WY, Lu PX, Assadi M, Huang XL, Skrahin A, Rosenthal A, Gabrielian A, Tartakovsky M, Wang YX. Updates on 18F-FDG-PET/CT as a clinical tool for tuberculosis evaluation and therapeutic monitoring. Quant Imaging Med Surg 2019;9:1132-46.

41. Jeon KN, Ha JY, Park MJ, Bae K, Baek HJ, Choi BH, Cho SB, Moon JI, Kim HC. Pulmonary Tuberculosis in Patients with Emphysema: Computed Tomography Findings. J Comput Assist Tomogr 2016;40:912-6.

42. Choo JY, Lee KY, Kim MY, Kang EY, Oh YW, Lee SH, Seo BK, Je BK. Pulmonary Tuberculosis Confirmed by Percutaneous Transthoracic Needle Biopsy: Analysis of CT Findings and Review of Correlations with Underlying Lung Disease. Balkan Med J 2014;31:208-13.

43. Takahashi M, Fukuoka J, Nitta N, Takazakura R, Nagatani Y, Murakami Y, Otani H, Murata K. Imaging of pulmonary emphysema: a pictorial review. Int J Chron Obstruct Pulmon Dis 2008;3:193-204.

44. Ogawa K, Kurosaki A, Miyamoto A, Takahashi Y, Murase K, Hanada S, Uruga H, Takaya H, Morokawa N, Kishi K. Clinicoradiological Features of Pulmonary Tuberculosis with Interstitial Pneumonia. Intern Med 2019;58:2443-9.

45. Shachor Y, Schindler D, Siegal A, Lieberman D, Mikulski $\mathrm{Y}$, Bruderman I. Increased incidence of pulmonary tuberculosis in chronic interstitial lung disease. Thorax 1989;44:151-3.

46. Chung MJ, Goo JM, Im JG. Pulmonary tuberculosis in patients with idiopathic pulmonary fibrosis. Eur J Radiol 2004;52:175-9.

47. Park SW, Song JW, Shim TS, Park MS, Lee HL, Uh ST, Park CS, Kim DS. Mycobacterial pulmonary infections in patients with idiopathic pulmonary fibrosis. J Korean Med Sci 2012;27:896-900.

48. Kligerman SJ, Franks TJ, Galvin JR. From the radiologic pathology archives: organization and fibrosis as a response to lung injury in diffuse alveolar damage, organizing pneumonia, and acute fibrinous and organizing pneumonia. Radiographics 2013;33:1951-75.

49. Oikonomou A, Hansell DM. Organizing pneumonia: the many morphological faces. Eur Radiol 2002;12:1486-96.

50. Zhao F, Yan SX, Wang GF, Wang J, Lu PX, Chen B, Yuan J, Zhang SZ, Wang YX. CT features of focal organizing pneumonia: an analysis of consecutive histopathologically confirmed 45 cases. Eur J Radiol 2014;83:73-8.

51. Kim YC, Jang AS, Kim JS, Oh SH, Lee YY, Son JG, Sun HJ, Lee JI. A case of pulmonary tuberculosis following bronchiolitis obliterans with organizing pneumonia (BOOP). Chonnam Med J 2002;38:170-4.

52. Yoon HS, Lee EJ, Lee JY, Chon GR, Lee SH, Kim SJ. Organizing pneumonia associated with Mycobacterium tuberculosis infection. Respirol Case Rep 2015;3:128-31.

53. Hsieh MH, Lin CY. Pulmonary tuberculosis presenting as organizing pneumonia. Am J Respir Crit Care Med 2014;189:e63.

Cite this article as: Zeng $Y$, Zhai XL, Wáng YXJ, Gao WW, $\mathrm{Hu}$ CM, Lin FS, Chai WS, Wang JY, Shi YL, Zhou XH, Yu HS, Lu XW. Illustration of a number of atypical computed tomography manifestations of active pulmonary tuberculosis. Quant Imaging Med Surg 2021;11(4):1651-1667. doi: 10.21037/ qims-20-1323 